\title{
A NOVEL SUBSPACE APPROACH FOR WIRELESS SENSOR NETWORK POSITIONING WITH RANGE MEASUREMENTS
}

\author{
Frankie K. W. Chan*, H. C. So*, and W.-K Ma ${ }^{\dagger}$ \\ * Department of Electronic Engineering, City University of Hong Kong, Tat Chee Avenue, Kowloon, Hong Kong \\ ${ }^{\dagger}$ Department of Electrical Engineering and Institute of Communications Engineering \\ National Tsing Hua University, Taiwan
}

\begin{abstract}
Estimating the positions of sensor nodes is a fundamental and crucial problem in wireless sensor networks. In this paper, a novel subspace approach for range-based measurements node localization is devised. Computer simulations are included to contrast the performance of the proposed algorithm with the conventional subspace positioning method, namely, classical multidimensional scaling, as well as theCramér-Rao lower bound.
\end{abstract}

Index Terms - subspace method, position estimation, wireless sensor networks

\section{INTRODUCTION}

Recent technological advances in wireless communications and microsystem integration have enabled the development of small, inexpensive, low-power sensor nodes to collect surrounding data, perform small-scale computations and communicate among their neighbors. These wirelessly connected nodes have numerous remote monitoring and control applications [1]-[3] such as asset management, habitat monitoring, health caring, building automation, battlefield surveillance, as well as environment observation and forecasting. Since sensor nodes are often arbitrarily placed with their positions being unknown, sensor positioning is a fundamental and crucial issue for the wireless sensor network (WSN) operation and management.

A number of node localization methods have been developed in the literature [4]-[11]. A straightforward technique is to exploit the connectivity information [4] - who is within the communication range of whom - to derive the node positions with the use of the anchor nodes subject to the proximity constraints imposed by the known connections. But it only provides coarse-grain location estimates. Apart from connectivity, range-based schemes which utilize nodeto-node or hop distances and/or angles can attain higher sensor positioning accuracy. The ranges are usually obtained from the pair-wise time-of-arrival (TOA), timedifference-of-arrival, received signal strength (RSS) and/or angle-of-arrival measurements, although it is also possible to use the average hop length and hop counts between indirectly connected nodes to deduce the distance information [5] as well. Assuming that the range measurements errors are Gaussian distributed, the maximum likelihood (ML) methods for node localization correspond to the nonlinear least squares problem [6]-[8]. The ML approach can attain optimum estimation performance but requires centralized data processing with intensive computations and sufficiently precise initial estimates for global convergence. On the other hand, suboptimal but computationally attractive range-based positioning techniques such as multilateration [9] and classical multidimensional scaling (MDS) [10]-[11] can allow distributed processing. In this paper, a computationally simple subspace algorithm for WSN positioning with the use of the node-to-node distance estimates deduced from the RSS or TOA measurements is devised.

The rest of the paper is organized as follows. The development of the subspace localization algorithm for WSN is shown in Section 2. Numerical examples are presented in Section 3 to compare the proposed algorithm with the MDS method [10] as well as CramérRao lower bound (CRLB) [12]. Finally, concluding remarks are given in Section 4.

\section{SUBSPACE POSITIONING APPROACH}

\section{II-A. Full Set Solution}

Consider a fully connected network of $M$ sensors in a two-dimensional space. (extension to the three- 
dimensional space is straightforward.) Let $\mathbf{s}_{i}=\left(x_{i}, y_{i}\right)$, $i=1,2, \cdots, M$, be the position of the $i$ th sensor. Without loss of generality, we assume that the positions of the first $k$ of them, $\mathbf{s}_{1}, \mathbf{s}_{2}, \cdots, \mathbf{s}_{k}$, where $k \geq 3$, are known. The task is to find $\mathbf{s}_{i}, i=k+1, k+2, \cdots, M$.

To derive our proposed positioning approach, we first let the distance between the $i$ th sensor and $j$ th sensor be $d_{i, j}$, given by:

$$
\begin{gathered}
d_{i, j}=d_{j, i}=\quad \sqrt{\left(x_{i}-x_{j}\right)^{2}+\left(y_{i}-y_{j}\right)^{2}}, \\
i, j=1,2, \cdots, M .
\end{gathered}
$$

Let ${ }_{m} \mathbf{C}_{n}=m ! /[n !(m-n) !]$, and consider a matrix $\mathbf{X} \in \mathbb{R}^{\left({ }_{M} \mathrm{C}_{2}-{ }_{k} \mathrm{C}_{2}\right) \times 2}$ which contains all the differences of $x_{i}-x_{j}$ and $y_{i}-y_{j}, i>j$, with at least one of the $\mathbf{s}_{i}$ and $\mathbf{s}_{j}$ being unknown. Mathematically, $\mathbf{X}$ is represented as

$\mathbf{X}=\left[\begin{array}{cc}x_{k+1} \mathbf{1}_{k}-\mathbf{x}_{k} & y_{k+1} \mathbf{1}_{k}-\mathbf{y}_{k} \\ x_{k+2} \mathbf{1}_{k+1}-\mathbf{x}_{k+1} & y_{k+2} \mathbf{1}_{k+1}-\mathbf{y}_{k+1} \\ \vdots & \vdots \\ x_{M} \mathbf{1}_{M-1}-\mathbf{x}_{M-1} & y_{M} \mathbf{1}_{M-1}-\mathbf{y}_{M-1}\end{array}\right]$

where

$$
\left[\begin{array}{c}
\mathbf{x}_{i}^{T} \\
\mathbf{y}_{i}^{T}
\end{array}\right]=\left[\begin{array}{llll}
x_{1} & x_{2} & \cdots & x_{i} \\
y_{1} & y_{2} & \cdots & y_{i}
\end{array}\right]
$$

with $\mathbf{1}_{i}$ and $(\cdot)^{T}$ denote the $i \times 1$ vector with all elements 1 and transpose operation, respectively. The matrix $\mathbf{X}$ can be expressed a linear combination of matrices with coefficients $x_{i}$ and $y_{i}, i=k+1, k+$ $2, \cdots, M$ :

$$
\mathbf{X}=\sum_{i=1}^{2(M-k)} \phi_{i} \mathbf{X}_{i}+\mathbf{X}_{0}
$$

where $\phi_{i}$ is the $i$ th element of the vector $\phi$ which is defined as

$\boldsymbol{\phi}=\left[\begin{array}{lllllll}x_{k+1} & y_{k+1} & x_{k+2} & y_{k+2} & \cdots & x_{M} & y_{M}\end{array}\right]^{T}$

Here, $\left[\mathbf{X}_{0}\right]_{i, j} \in\left\{0,-x_{1},-y_{1},-x_{2},-y_{2}, \cdots,-x_{k},-y_{k}\right\}$, $\left[\mathbf{X}_{k}\right]_{i, j} \in\{-1,0,1\}$ and $[\mathbf{X}]_{i, j}$ denotes the $(i, j)$ entry of $\mathbf{X}$.

The subspace method to be presented is based on examining the subspace and rank properties of the socalled multidimensional similarity matrix [13]

$$
\mathbf{D}=\mathbf{X X}^{T}
$$

The rank of $\mathbf{D}$ is $\operatorname{rank} \mathbf{D}=\operatorname{rank} \mathbf{X}=2$. The matrix $\mathbf{D}$ is constructed from $\left\{d_{i, j}\right\}$ using the following formula:

$$
\mathbf{D}=-0.5 \mathbf{K R K}^{T}
$$

where

$$
\begin{aligned}
{[\mathbf{R}]_{i, j} } & =d_{i, j}^{2} \\
\mathbf{K} & =\left[\begin{array}{llll}
\mathbf{E}_{k+1} & \mathbf{E}_{k+2} & \cdots & \mathbf{E}_{M}
\end{array}\right]^{T} \\
\mathbf{E}_{i} & =\left[\begin{array}{llll}
\mathbf{e}_{i, 1} & \mathbf{e}_{i, 2} & \cdots & \mathbf{e}_{i, i-1}
\end{array}\right] \\
& =\left[\begin{array}{lll}
-\mathbf{I}_{i-1} & \mathbf{1}_{i-1} & \mathbf{0}_{(i-1) \times(M-i)}
\end{array}\right]^{T}, \\
i & =k+1, k+2, \cdots, M
\end{aligned}
$$

and

$$
\mathbf{e}_{i, j}=\mathbf{e}_{i}-\mathbf{e}_{j}
$$

with $\mathbf{0}_{i \times j}$ and $\mathbf{e}_{i}$ denote respectively, the $i \times j$ zero matrix and the $i$ th column of $\mathbf{I}_{M}$. Since $\mathbf{D}$ is symmetric, positive semidefinite, and of rank 2 , it can be decomposed using eigenvalue factorization as

$$
\mathbf{D}=\mathbf{U}_{s} \boldsymbol{\Lambda}_{s} \mathbf{U}_{s}^{T}+\mathbf{U}_{n} \boldsymbol{\Lambda}_{n} \mathbf{U}_{n}^{T}
$$

where $\boldsymbol{\Lambda}_{s}=\operatorname{diag}\left(\lambda_{1}, \lambda_{2}\right) \succ \mathbf{0}, \boldsymbol{\Lambda}_{n}=$ $\operatorname{diag}\left(\lambda_{3}, \cdots, \lambda_{M} \mathrm{C}_{2}-{ }_{k} \mathrm{C}_{2}\right)$ with $\lambda_{3}=\lambda_{4}=\cdots=$ $\lambda_{M} \mathrm{C}_{2}-{ }_{k} \mathrm{C}_{2}=0$ and the columns of $\mathbf{U}_{s} \in$ $\mathbb{R}^{\left({ }_{M} \mathrm{C}_{2}-{ }_{k} \mathrm{C}_{2}\right) \times 2}$ and $\mathbf{U}_{n} \in \mathbb{R}^{\left({ }_{M} \mathrm{C}_{2}-{ }_{k} \mathrm{C}_{2}\right) \times\left({ }_{M} \mathrm{C}_{2}-{ }_{k} \mathrm{C}_{2}-2\right)}$ are called the signal and noise eigenvectors, respectively. As $\mathbf{X}$ lies in the signal subspace $\mathbf{U}_{s}$, we have

$$
\mathbf{U}_{n}^{T} \mathbf{X}=\mathbf{0}_{\left({ }_{M} \mathrm{C}_{2}-{ }_{k} \mathrm{C}_{2}-2\right) \times 2}
$$

or by (3)

$$
\sum_{i=1}^{2(M-k)} \phi_{i} \mathbf{U}_{n}^{T} \mathbf{X}_{i}=-\mathbf{U}_{n}^{T} \mathbf{X}_{0} .
$$

When disturbance is present, noise-free distances $\left\{d_{i, j}\right\}$ are available only for $k \geq i>j \geq 1$. In our study, we generalize all the observed distance measurements, denoted by $r_{i, j}$, as

$$
r_{i, j}=r_{j, i}=d_{i, j}+q_{i, j}
$$

where $q_{i, j}=q_{j, i}$ is the possible zero-mean noise with variance $\sigma_{i, j}^{2} \geq 0$ if $\max (i, j)=k+1, k+2, \cdots, M$, and $\sigma_{i, j}^{2}=0$ otherwise with $\max (i, j)=i$ if $i \geq j$. This means that for practical purposes, $\left\{r_{i, j}\right\}$ will be substituted for $\left\{d_{i, j}\right\}$ in (5) when the latter is unavailable in forming $\mathbf{D}$. As a result, the equal signs in (7) and (8) should be replaced by the approximate 
equal signs and $\lambda_{3} \geq \lambda_{4} \geq \cdots \geq \lambda_{M} \mathrm{C}_{2}-{ }_{k} \mathrm{C}_{2} \geq 0$. Taking vectorization on both sides of (8) yields

$$
\mathbf{A} \phi \approx \mathbf{b}
$$

where

$\mathbf{A}=\left[\begin{array}{lll}\operatorname{vec}\left(\mathbf{U}_{n}^{T} \mathbf{X}_{1}\right) & \cdots & \operatorname{vec}\left(\mathbf{U}_{n}^{T} \mathbf{X}_{2(M-k)}\right)\end{array}\right]$

and

$$
\mathbf{b}=-\operatorname{vec}\left(\mathbf{U}_{n}^{T} \mathbf{X}_{0}\right)
$$

with $\operatorname{vec}(\cdot)$ being the vectorization operator. A simple and straightforward estimate of $\phi$, denoted by $\hat{\phi}$, is obtained by least squares (LS):

$$
\hat{\phi}=\mathbf{A}^{\dagger} \mathbf{b}
$$

where $\mathbf{A}^{\dagger}=\left(\mathbf{A}^{T} \mathbf{A}\right)^{-1} \mathbf{A}^{T}$ is the pseudo-inverse of $\mathbf{A}$. We refer this subspace based estimate to as the full-set solution because all admissible pairs in $\left\{\mathbf{s}_{i}\right\}$ have been employed.

\section{II-B. Minimal Set Solution}

In fact, the computational complexity of our proposed approach can be significantly reduced by considering the rank deficiency of $\mathbf{D}$ as follows. From (5), it can be shown that

$$
\begin{aligned}
& \mathbf{E}_{i+1}\left[\begin{array}{ll}
\mathbf{I}_{i-1} & -\mathbf{1}_{i-1}
\end{array}\right]^{T}=\mathbf{E}_{i}, i=k+1, \cdots, M-1 \\
& \Rightarrow \operatorname{span}\left(\mathbf{E}_{k+1}\right) \subset \operatorname{span}\left(\mathbf{E}_{k+2}\right) \subset \cdots \subset \operatorname{span}\left(\mathbf{E}_{M}\right) \\
& \Rightarrow \operatorname{rankK}=\operatorname{rank} \mathbf{E}_{M}=M-1
\end{aligned}
$$

where $\operatorname{span}(\mathbf{A})$ is the space spanned by the columns of A. Therefore, the dimension of $\mathbf{D}$ can be significantly reduced to $(M-1)$. For example, we can choose

$$
\begin{aligned}
\breve{\mathbf{D}} & =\breve{\mathbf{X}} \breve{\mathbf{X}}^{T} \\
& =-0.5 \mathbf{E}_{M-1}^{T} \mathbf{R E}_{M-1}
\end{aligned}
$$

where

$$
\breve{\mathbf{X}}=\left[\begin{array}{llll}
x_{M}-x_{1} & x_{M}-x_{2} & \cdots & x_{M}-x_{M-1} \\
y_{M}-y_{1} & y_{M}-y_{2} & \cdots & y_{M}-y_{M-1}
\end{array}\right]^{T}
$$

or any $(M-1)$ rows of $\mathbf{K}$ such that $\operatorname{rank} \breve{\mathbf{D}}=M-1$. It should be noted that the choice of $\breve{\mathbf{D}}$ is not unique because different rows of $\mathbf{K}$ can be selected as long as the rank requirement is satisfied. Moreover, different choices may give different estimation accuracy. The position estimate of this simpler scheme follows the same procedure of (6)-(12) and we refer it to as the minimum set solution.

\section{NUMERICAL EXAMPLES}

Computer simulation has been conducted to compare the mean square position errors (MSPEs) of the subspace algorithm with the classical MDS method [10] as well as CRLB [12] for WSN localization. The additive noise $q_{i, j}$ is a zero-mean white Gaussian process. The signal-to-noise ratio (SNR) of all range measurements is set to be identical and it is defined as $\mathrm{SNR}=d_{i, j}^{2} / \sigma_{i, j}^{2}$. A WSN of 14 fully-connected sensors is considered with 10 of them begin unknown. The 14 sensors are located in a $100 \times 200 \mathrm{~m}^{2}$ area and we position the anchor sensors at $(0,0) \mathrm{m},(0,100) \mathrm{m}$ $(100,0) \mathrm{m}$ and $(100,100) \mathrm{m}$. All simulation results are averages of 1000 independent runs.

In the first scenario, the 10 unknown sensors have fixed positions and the WSN geometry is shown in Figure 1. The average of the MSPEs for the 10 unknown sensors is plotted in Figure 2 to illustrate the overall performance. It can be observed that both the full set and minimum set subspace solutions have smaller MSPEs than those of the MDS method, though they are inferior to the CRLB by about $8 \mathrm{dBm}^{2}$ and $11 \mathrm{dBm}^{2}$, respectively, in the whole SNR range.

In the second scenario, the positions of the 10 unknown sensors are located randomly according to a uniform distribution in the $100 \times 200 \mathrm{~m}^{2}$ area while the anchor positions remain unchanged in each independent run. Comparing with the first test, both the proposed subspace methods and MDS have larger MSPEs but the former is still superior to the latter by around $3.5 \mathrm{dBm}^{2}$ and $1 \mathrm{dBm}^{2}$, respectively. The results indicate that the proposed subspace approach outperforms the MDS solution for various sensor geometries in an average sense.

\section{CONCLUSION}

Two novel subspace based solutions have been devised for node localization in fully-connected wireless sensor networks (WSNs) using distance measurements. Although the proposed positioning approach gives suboptimum estimation performance, it is superior to the classical multidimensional scaling scheme. Future research will be focused on modifying the proposed methodology so that it can be operated in a distributed manner and/or partially-connected WSNs.

\section{ACKNOWLEDGEMENT}

This work described in this paper was fully supported by a grant from the Research Grants Council of 
the Hong Kong Special Administrative Region, China (Project No. CityU 119606).

\section{REFERENCES}

[1] A. Hać, Wireless Sensor Network Designs, NJ : John Wiley, 2003

[2] M. Ilyas and I. Mahgoub, Handbook of Sensor Networks: Compact Wireless and Wired Sensing Systems, London: CRC Press, 2005

[3] H. Karl and A. Willig, Protocols and Architectures for Wireless Sensor Networks, NJ: Wiley, 2005

[4] L. Doherty, K S.J. Pister, L. El Ghaoui, "Convex position estimation in wireless sensor networks," Proc. IEEE INFOCOM, pp.1655-1663, 2001

[5] D. Niculescu and B. Nath, "Ad hoc positioning system (APS)," Proc. IEEE GlobeCom, vol.5, pp.2926-2931, San Antonio, AZ, Nov. 2001

[6] R.L. Moses, D. Krishnamurthy and R.M. Patterson, "A self-localization method for wireless sensor networks," EURASIP Journal on Applied Signal Processing, vol.4, pp.348-358, 2003

[7] N. Patwari, A.O. Hero, III, M. Perkins, N.S.Correal and R.J. O'Dea, "Relative location estimation in wireless sensor networks," IEEE Transactions on Signal Processing, vol.51, no.8, pp.2137-2148, August 2003

[8] M. Meurer, P.W. Baier, T. Weber, C.A. Jotten and S. Heilmann, "SPIDER: enhanced distance based localization of mobile radio terminals," Proc. VTC 2004Fall, vol.5, pp.3521-3525, Sept. 2004

[9] A. Savvides, C.-C. Han and M.B. Srivastava, "Dynamic fine-grained localization in ad-hoc networks of sensors," Proc. 7th Annual Int. Conf. Mobile Computing and Networking, pp.166-179, ACM Press, Rome, Italy, July 2001

[10] X. Ji and H. Zha, "Robust sensor localization algorithm in wireless ad-hoc sensor networks," Proc.Computer Communications and Networks, pp. 527-532, Oct. 2003.

[11] Y. Shang, W. Ruml, Y. Zhang and M. Fromherz, "Localization from connectivity in sensor networks," IEEE Trans. Parallel and Distributed Systems, vol.15, pp.961-974, Nov. 2004

[12] N. Patwari, J.N. Ash, S. Kyperountas, A.O. Hero, III, R.L. Moses and N.S. Correal, "Locating the nodes: cooperative localization in wireless sensor networks," IEEE Signal Processing Magazine, pp.54-69, July 2005

[13] Q. Wan, Y.-J. Luo W.-L. Yang, J. Xu, J. Tang and Y.-N. Peng, "Mobile localization method based on multidimensional similarity analysis," Proc. ICASSP 2005, vol.IV, pp.1081-1084, Phoenix, USA

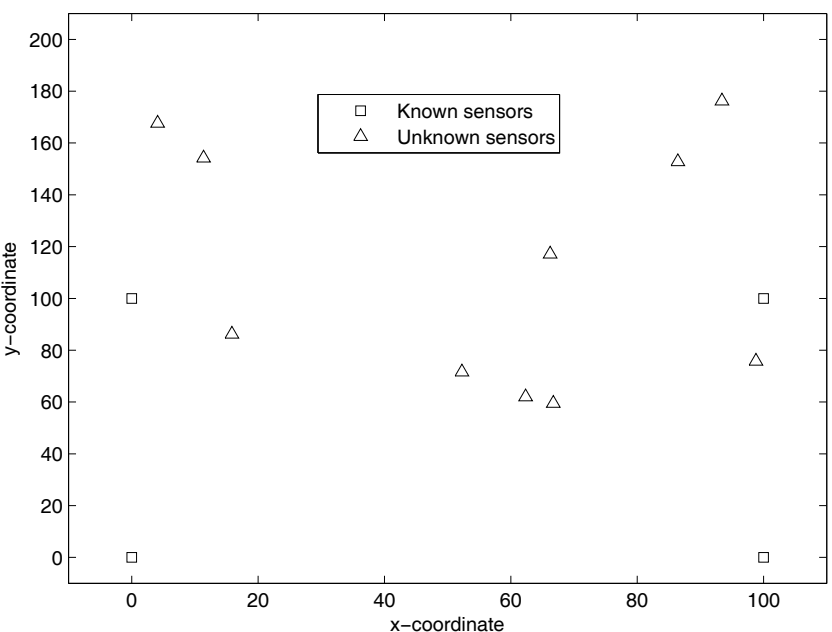

Fig. 1. Positions of the sensors

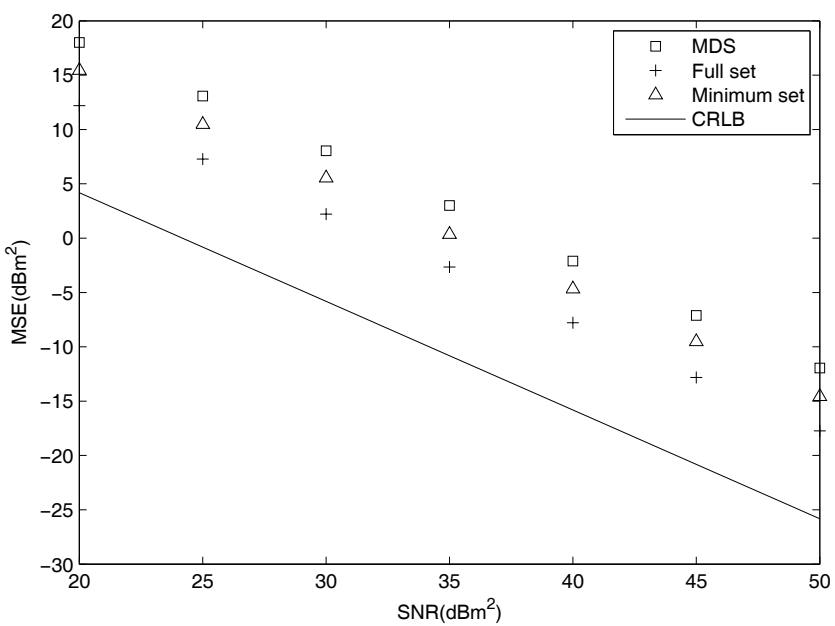

Fig. 2. Mean square position error versus SNR with fixed-position sensors

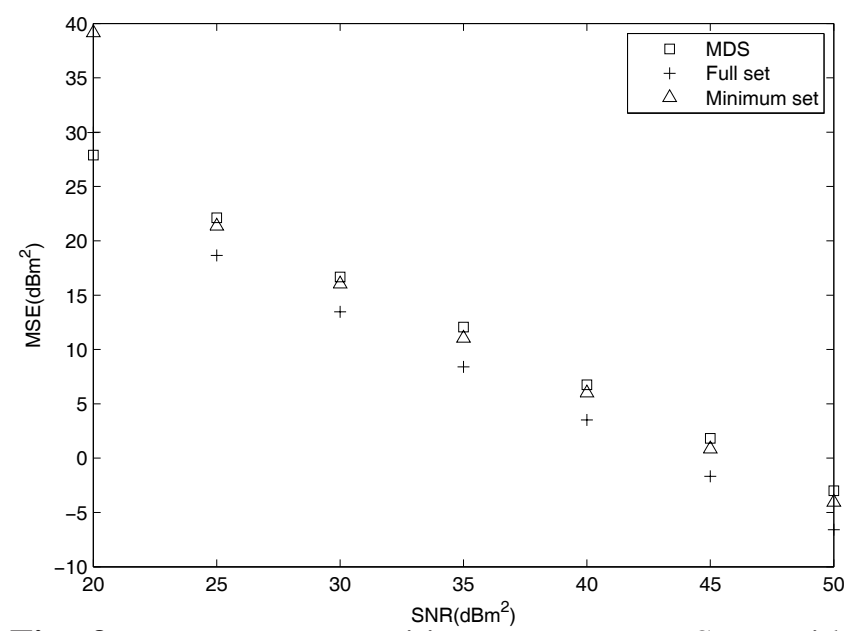

Fig. 3. Mean square position error versus SNR with random-position sensors 